\title{
Long-range order model of aqueous electrolyte solutions
}

\author{
Ricardo Marcos Pytkowicz, Kenneth Johnson and Cynthia Curtis \\ School of Oceanography, Oregon State University, Corvallis, Oregon 97331, U.S.A.
}

(Received December 15, 1976)

\begin{abstract}
A partial long-range order model for aqueous electrolyte solutions is proposed to avoid contradictions present in the Debye-Hückel theory. The partial long-range order increases with increasing salt concentration because, as the ions are closer together, the coulombic energy of interaction which generates a quasi-lattice increases. Furthermore, the order decreases with increasing temperature because the thermal energy increases relative to the coulombic attraction of the ions. The long-range order parameter $\mathrm{L}$ and a parameter $\mathrm{v}$ which results from fluctuations and other effects are obtained for $\mathrm{LiCl}, \mathrm{NaCl}$ and $\mathrm{KCl}$ by comparing the theoretical configurational free energy to the electrical free energy obtained from the experimental activity coefficients. The specific interaction and the cluster integral models are discussed briefly at the end of this work.
\end{abstract}

\section{INTRODUCTION}

Activity coefficients of electrolytes are important to understand ionic interactions and, therefore, the properties of ocean and lake waters, interstitial waters and other solutions of geochemical interest. Although activity coefficients can be measured, an insight into their meaning provides a solid theoretical foundation for the study of natural waters.

Several approaches such as ion-pair models and specific interaction ones have been applied to geochemical solutions and these approaches have at times required the use of the extended Debye-Hückel equation (1923) or of its simplified form

$$
\log f_{ \pm}=\frac{A I^{0.5}}{1+I^{0.5}}
$$

where $f_{ \pm}$represents the mean activity coefficient, $\overline{\mathrm{A}}$ is the Debye constant and I represents the ionic strength.

We will show that the Debye-Hückel theory is inconsistent at intermediate ionic strength and will suggest a model which avoids the contradiction present in their theory. In the future, we plan to extend our model to mixed electrolyte solutions to seek an understanding of solutions such as seawater.

Debye-Hückel theory; a critique The DebyeHückel theory yields a net charge density $\rho_{(\mathrm{J})}$ around a central cation $\mathbf{J}$ given by

$$
\rho_{(\mathrm{J})}=\frac{\mathrm{Z}_{\mathrm{J}} \epsilon \kappa^{2} \mathrm{e}^{\kappa \mathrm{a}_{\mathrm{D}}}}{4 \Pi 1+\kappa \mathrm{a}_{\mathrm{D}}} \frac{\mathrm{e}^{-\kappa \mathrm{r}(\mathrm{J})}}{\mathrm{r}_{\mathrm{J}}}
$$

where $z_{\mathbf{J}}$ is the valence of $J, \epsilon$ is the electronic charge, $\kappa$ is the reciprocal of the thickness of the ion-atmosphere, $a_{D}$ is the Debye radius, and $r_{(J)}$ is the distance from $J$. One notes that $\rho_{(\mathrm{J})}$ decreases monotonically from a maximum value at $r_{J}=a_{D}$ (the surface of $J$ ) to zero at $r=\infty$. This equation represents the ion-cloud of $\mathrm{J}$.

It can be seen from the equations of Debye and Hückel that the total potential (due to $\mathrm{J}$ and to the ion cloud) also decreases monotonically with the distance from $J$. The ion cloud, however, must be fine-grained (FRANK and THOMPSON, 1959), that is, there are many ions within a distance $1 / \kappa$ from $J$. The potential should, therefore, oscillate with increasing $r_{(J)}$ instead of decreasing steadily throughout the cloud.

Another difficulty arises because the potential $\phi_{(\mathrm{J})}$ due to the effect of all the ions in solution upon a volume element $\mathrm{dV}$ situated at a distance $r_{(J)}$ from the central ion is not uniquely defined as a function of position. $\phi_{(\mathrm{J})}$ is given by

$$
\phi_{(\mathrm{J})}=\frac{\mathrm{z}_{\mathrm{J}} \epsilon}{\mathrm{D}_{\mathrm{e}}} \times \frac{\mathrm{e}^{\kappa \mathrm{a}_{\mathrm{D}}}}{1+\kappa \mathrm{a}_{\mathrm{D}}} \times \frac{\mathrm{e}^{-\kappa \mathrm{r}(\mathrm{J})}}{\mathrm{r}_{(\mathrm{J})}}
$$


where $\mathrm{D}_{\mathrm{e}}$ is the dielectric constant.

If we select two like ions, $\mathrm{J}-1$ and $\mathrm{J}-2$ successively as the central ion but retain the volume element $\mathrm{dV}$ fixed, the potentials due to all ions in the solution, $\phi_{(\mathrm{J}-1)}$ and $\phi_{(\mathrm{J}-2)}$, at $\mathrm{dV}$ will be different as $r_{(\mathrm{J}-1)}$ and $\mathrm{r}_{(\mathrm{J}-2)}$ are different. Thus, we see that $\phi_{(J)}$ is indeed not uniquely determined as a function of position in the solution.

The problems described above arise primarily because Debye and Hückel used the Boltzman law

$$
n_{i}^{\prime}=n_{i} \exp \left(-\frac{z_{i} \epsilon \phi(J)}{k_{B} T}\right)
$$

for the distribution of ions around the central ion J. $n_{i}^{\prime}$ is the number of ions of type i per unit volume near $J, n_{i}$ is the bulk value far from $\mathrm{J}, \mathrm{k}_{\mathrm{B}}$ is Boltzman's constant, and $\mathrm{T}$ is the absolute temperature. The Boltzman law can only be applied rigorously to non-interacting particles or to particles that interact solely through elastic collisions which do not affect the free energy of the system.

The success of the Debye-Hückel limiting law at very high dilutions is explainable because in this case the ionic interactions are very weak and the Boltzman law is a valid approximation. The success of their extended equation

$$
\log \mathrm{f}_{ \pm}=-\frac{\mathrm{AI}^{0.5}}{1+\mathrm{Ba}_{\mathrm{D}} \mathrm{I}^{0.5}}
$$

however, results from the use of $a_{D}$ as an empirical curve fitting parameter.

\section{First APPROXimation to a LatTice MODEL Perfect Long-Range Order}

We will first examine the hypothetical case of perfect long-range order and later consider the real case of partial order for greater clarity. We should mention that FRANK and THOMPSON (1959) and BAHE (1972) considered the possibility of ionic short-range order. We prefer long-range order because there is no a priori reason to expect the time-average environment of any ion of type $\mathrm{i}$ to be different from that of any other $\mathrm{i}$ ion and because, as we will demonstrate, a minimum free-energy can be found as a function of the long-range order parameter $\mathrm{L}$ with $\mathrm{L}>0$. It is only when the minimum occurs at $\mathrm{L}=0$ (long-range randomness) that short-range order need be considered
(KITTEL, 1959).

It may appear at first glance that long-range order does not make sense in a fluid that may be in motion. One can reason around this problem as follows. Water contains molecules in a hydrogen-bonded structure and, if these coherent domains when in flow last longer than the time required for the rearrangement of ions and are large enough so that the properties of their surfaces can be neglected, then a lattice theory can be used. This is the case because from the lattice theory one obtains the configurational free energy $\Delta F_{L}$, enthalpy $\Delta H_{L}$, and entropy $\Delta \mathrm{S}_{\mathrm{L}}$ and these are extensive properties which can be added for the coherent domains provided that the domain surfaces can be neglected. It is easy to show that surfaces are negligible if the average domain sizes are roughly larger than 0.1 micron. Then, longrange order leads to $\Sigma \Delta F_{L, d}$, summed over all the domains $d$, which is the same as the $\Delta F_{L}$ which would result from long-range order in the whole solution.

We have observed that activity coefficients determined with electrodes do not appear to depend upon the rate of stirring and, furthermore, agree with results from static isopiestic measurements, indicating that coherent domains with the desired properties may indeed exist.

Let us consider the cohesive energy of an ionic crystal (e.g., Pauling, 1960; KitTel, 1959) before proceeding to solutions. This energy is

$$
E_{(x t a l)}=-\frac{N z^{2} \epsilon^{2} A_{M}}{r_{o}}+\frac{B_{b}}{r_{o}{ }^{n}}
$$

$\mathrm{N}$ is the number of ions of each type, $A_{M}$ is the Madelung constant, $r_{o}$ is the nearest neighbor distance, and the last term represents the Born repulsion.

In the case of an hypothetical solution in which the ions are perfectly ordered, that is, they occupy the centers of equal cubes with cations and anions alternating in consecutive cubes (face-centered cubic lattice), we can express the configurational energy by

$$
\Delta E_{L}=-\frac{N_{A} z^{2} \epsilon^{2} A_{M}}{D_{e} r_{o}} m
$$

$\mathrm{N}_{\mathrm{A}}$ is Avogadro's number and $\mathrm{m}$ is the molality. The repulsion term of equation (6) can be neglected except for very high concentrations. As 


$$
\mathrm{r}_{\mathrm{o}}=\left[\frac{1000+\mathrm{mM}^{1 / 3}}{2 \mathrm{~N}_{\mathrm{A}} \mathrm{m} \rho}\right]^{1 / 3}
$$

equation (7) becomes

$$
\Delta \mathrm{E}_{\mathrm{L}}=\frac{\mathrm{B}_{\mathrm{L}} \mathrm{z}^{2} \mathrm{~A}_{\mathrm{M}} \rho^{1 / 3}}{(1+0.001 \mathrm{mM})^{1 / 3}} \mathrm{~m}^{4 / 3}
$$

where $\rho$ is the solution density, $\mathrm{M}$ is the molecular weight of the solute and

$$
\mathrm{B}_{\mathrm{L}}=\frac{2^{1 / 3} \mathrm{~N}_{\mathrm{A}}^{4 / 3} \epsilon^{2}}{10 \mathrm{D}_{\mathrm{e}}}
$$

$\Delta E_{L}$ is a configurational enthalpy but, by neglecting the electrostriction, it can be considered an electrical internal energy. There is also a configurational entropy term $\Delta \mathrm{S}_{\mathrm{L}}$ but, to save space, it will be entered when we discuss the partial order model.

\section{Partial Long-Range Order Model}

It would be surprising indeed if ions were perfectly ordered when in solution as the interactions between cations and anions are much weaker in solution than in the crystal due to the large dielectric constant of water and to the greater interionic spacing. From the perfect order model, one obtains a $1 / 3$ power concentration dependence of $\log \mathrm{f}_{ \pm}$(RoBINSON and Stokes, 1959; PYTKowicz et al., 1976), where $\mathrm{f}_{ \pm}$is the mean activity coefficients, which does not correspond to the observed concentration dependence.

PYTKOWICZ et al. (1976) proposed a partial long-range order which increases with concentration and decreases with temperature. The increase with concentration occurs because the energy of ionic interaction, which yields the quasi-lattice, increases as the ions become closer together. The decrease with increasing temperature results from the enhanced thermal motion.

In order to calculate the configurational free energy rigorously as a function of the degree of order, PYTKOWICZ et al. employed the following artifice. Let $w$ represent the fraction of wrong ions, that is, of cations in the anionic sub-lattice and vice versa. Let us set $\mathrm{w}=0.25$ to clarify our thoughts. The time-average configuration of what is in reality a flickering situation is shown in Fig. 1. We obtain a perfectly ordered lattice in which to each cation we
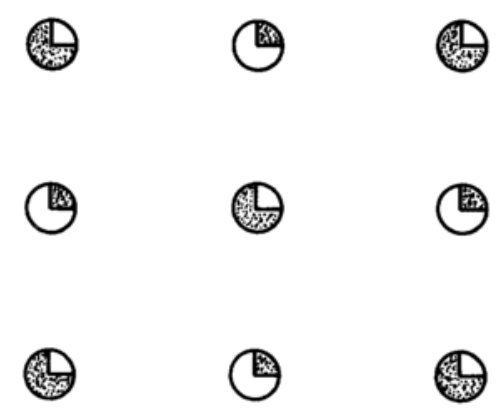

\section{Pure Cation}

\section{Pure Anion}

Fig. 1. Partial long-range order for $w=0.25$.

ascribe $25 \%$ anionic character and vice versa. The ion charge is now $\pm z \epsilon(1-2 \mathrm{~W})$ and, if we set $(1-2 w)=L$, equation (9) becomes

$$
\Delta E_{L}=-\frac{B_{L} z^{2} A_{M} \rho^{1 / 3} L^{2} v}{(1+0.001 M)^{1 / 3}} m^{4 / 3}
$$

$\mathrm{L}$ is a convenient long-range order parameter because $\mathrm{w}=0.5$ yields $\mathrm{L}=0$ (randomness) and $\mathrm{w}=0$ results in $\mathrm{L}=1$. $\mathrm{L}$ is expected to increase with increasing $\mathrm{m}$ due to the greater energy of interaction relative to $\mathrm{kT}$ and to decrease with increasing temperature. The term $\mathrm{v}$ was introduced in anticipation of what follows.

Let us consider the simple case of two fixed anions separated by a distance $2 r_{o}$ and a cation fluctuating around its central position, as is shown in Fig. 2. If the cation is displaced either to the right or to the left by a distance $\mathrm{x}$ then its mean distance from the anions appears in the energy of interaction as

$$
r_{x}=0.5\left(\frac{1}{r_{o}-x}+\frac{1}{r_{o}+x}\right)=\frac{r_{o}}{r_{o}^{2}-x^{2}}>\frac{1}{r_{o}}
$$

Thus, fluctuations enhance the configurational energy. For every state set by a pair of values $(\mathrm{T}, \mathrm{m})$ one expects an average value $\bar{r}_{\mathrm{x}}$ for the lattice. $\quad \bar{r}_{x} / r_{o}$ should increase towards unity as $\mathrm{m}$ increases because $\Delta \mathrm{E}_{\mathrm{L}}$, which tends to fix ions in their lattice sites, becomes larger relative to the thermal energy.

Therefore, $v$ consists of two terms; $r_{o} / \bar{r}_{x}$ and a term $v^{\prime}$ which may include the charge in dielectric constant, ion-cavity interactions 


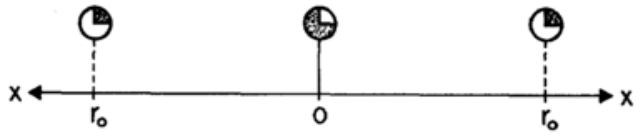

Fig. 2. Fluctuation model.

(DESNOYERS and CoNWAY, 1964), hard-core repulsive effects (PITZER, 1973), ion-cavity interactions (BAHE, 1972) and ion-pairing (PYTKOWICZ and KESTER, 1969).

The configurational free energy is $\Delta \mathrm{F}_{\mathrm{L}}=$ $\Delta E_{L}-T \Delta S_{L}$ if electrostriction is neglected. The term $\Delta S_{L}$ can be calculated by analogy to the case of binary solutions, as shown for example by KITTEI (1959) and $\Delta \mathrm{F}_{\mathrm{L}}$ is found to be, when $r_{0}$ is expressed in terms of $m$,

$$
\begin{aligned}
\Delta \mathrm{F}_{\mathrm{L}}= & -\frac{\mathrm{B}_{\mathrm{L}} \mathrm{z}^{2} \mathrm{~A}_{\mathrm{M}} \rho^{1 / 3} \mathrm{~L}^{2} \mathrm{v}}{\left(1+0.001 \mathrm{mM}_{2}\right)^{1 / 3}} \\
& +\mathrm{RTm}[(1+\mathrm{L}) \ln (1+\mathrm{L}) \\
& +(1-\mathrm{L}) \ln (1-\mathrm{L})]
\end{aligned}
$$

\section{Calculation of L and v: Theory}

The value of $\Delta F_{L}$ that corresponds to the configurational free energy obtained from experiments $\Delta \mathrm{F}_{\text {exp }}$ must obey two conditions. Firstly, $\Delta \mathrm{F}_{\mathrm{L}}$ at each given $\mathrm{m}$ must be a minimum relative to $\mathrm{L}$ in order to represent the most stable configuration. This condition is obeyed when

$$
\frac{1}{\mathrm{LV}} \ln \frac{1+\mathrm{L}}{1-\mathrm{L}}=\frac{2 \mathrm{~B}_{\mathrm{L}} \mathrm{z}^{2} \mathrm{~A}_{\mathrm{M}} \rho^{1 / 3} \mathrm{vm}^{1 / 3}}{\mathrm{RT}\left(1+0.001 \mathrm{mM}_{2}\right)^{1 / 3}}
$$

which is obtained by setting $\partial \Delta \mathrm{F}_{\mathrm{L}} / \partial \mathrm{L}=0$. The second condition is that

$$
\Delta \mathrm{F}_{\mathrm{L}}=\Delta \mathrm{F}_{\exp }
$$

where $\Delta \mathrm{F}_{\text {exp }}$ is obtained from measured activity coefficients.

At this point, we have two choices which will be illustrated with reference to Fig. 3, in which $n_{w}$ and $n_{s}$ are the numbers of moles of water and of salt. The total free energy $\Delta \mathrm{F}$ of mixing $55.5=\mathrm{n}_{\mathrm{w}}$ moles of water with $\mathrm{n}_{\mathrm{s}}=$ $\mathrm{m}$ moles of salt is given by

$$
\Delta \mathrm{F}=55.5 \mu_{\mathrm{w}}+\mathrm{m} \mu_{\mathrm{s}}=\Delta \mathrm{F}_{\mathrm{w}}+\Delta \mathrm{F}_{\mathrm{s}}
$$

where $\mu_{\mathrm{w}}$ and $\mu_{\mathrm{s}}$ are the chemical potentials

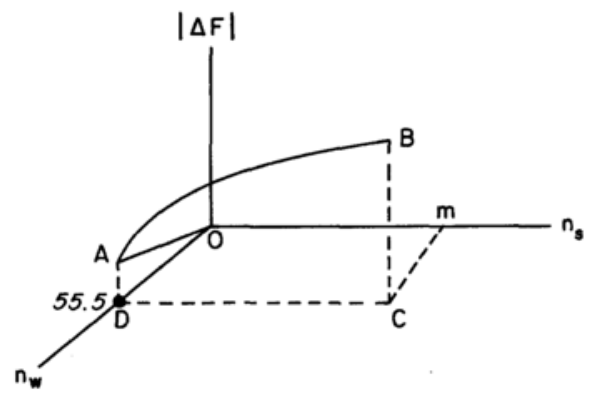

Fig. 3. Free energy of formation of a m molal solution.

of the water and of the salt and $F_{w}$ amd $F_{s}$ are the respective free energies and $\Delta \mathrm{F}$ corresponds to the length of the line segment $\overline{\mathrm{BC}}$. Physically, equation (8) would correspond to adding premixed solution of molality $\mathrm{m}$ to the container until $1,000 \mathrm{~g}$ of water and $n_{s}=m$ moles of salt were present in it.

Most measurements, however, follow the path OAB. The path OA corresponds to adding $1 \mathrm{~kg}$ of pure water to the container so that the length $\overline{\mathrm{AD}}$ is simply

$$
\Delta \mathrm{F}_{\mathrm{w}}^{\circ}=55.5 \mu_{\mathrm{w}}^{\circ}
$$

The path $\mathrm{AB}$ yields a free energy corresponding to the length $\overline{\mathrm{BC}}-\overline{\mathrm{AD}}$ which is

$$
\begin{aligned}
\Delta \mathrm{F}_{\mathrm{s}, \mathrm{w}} & =\int_{0}^{\mathrm{m}}\left(\begin{array}{c}
\partial \mathrm{F} \\
\partial \mathrm{n}_{\mathrm{s}}
\end{array}\right)_{\mathrm{P}, \mathrm{T}, \mathrm{n}_{\mathrm{w}}} \mathrm{dn}_{\mathrm{s}}=\int_{\mathrm{\rho}}^{\mathrm{m}}(\mathrm{dF})_{\mathrm{P}, \mathrm{T}, \mathrm{n}_{\mathrm{w}}}= \\
& =\int_{0}^{\mathrm{m}} \mathrm{dF}_{\mathrm{w}}+\mathrm{dF}_{\mathrm{s}}^{\mathrm{m}}
\end{aligned}
$$

where $F$ represents the free energy. $\mathrm{dF}_{\mathrm{w}}$, the change in the free energy of the water, appears even though $\mathrm{n}_{\mathrm{w}}=55.5=$ constant because when salt is added the composition changes and, therefore, the intensive quantities $\mu_{\mathrm{w}}$ and $\mu_{\mathrm{s}}$ which are not independent of each other change.

Now, $\mathrm{dF}_{\mathrm{w}}=55.5 \mathrm{RTd} \ln \mathrm{a}_{\mathrm{w}}$ and when $\mathrm{m}=$ 0 we have $a_{w}$, the activity of water, equal to zero. Therefore,

$$
\int_{0}^{\mathrm{m}} \mathrm{dF}_{\mathrm{w}}=55.5 \mathrm{RT} \ln \mathrm{a}_{\mathrm{w}}
$$




$$
\begin{aligned}
\int_{0}^{\mathrm{m}} \mathrm{dF}_{\mathrm{s}}= & \Delta \mathrm{F}_{\mathrm{s}}=\mathrm{m} \mu_{\mathrm{s}}=\mathrm{m}\left(\mu_{\mathrm{s}}^{\circ}+2 \mathrm{RT} \ln \mathrm{f}_{ \pm \mathrm{s}}\right. \\
& +\mathrm{RT} \ln \mathrm{m})
\end{aligned}
$$

Adding equations (17), (19), and (20) one obtains equation (16), that is, the total free energy of the solution if the states of the water and of the salt previous to bringing them to the container are not taken into consideration.

Note that $m \mu_{\mathrm{s}}^{\circ}$ contains the 'ideal' energy of hydration, that is the energy of hydration for a hypothetical system containing $\mathrm{m}$ moles of salt which acts as if it were present at infinite dilution. Thus, the 'ideal' binding of water of hydration does not affect the concentration of free water so that $a_{w}=1$. Equation (19) represents the change in the ratio $\rho_{\mathrm{w}}{ }^{*} / \rho_{\mathrm{w}}{ }^{* 0}$ of the fugacities of water in the solution and in pure water due to the actual binding of the water of hydration and to the randomization of water molecules next to the hydrated ones when the solute is present at a finite concentration.

One has, therefore, the ideal free energy

$$
\begin{aligned}
\Delta \mathrm{F}_{\mathrm{i}}=55.5\left(\mu_{\mathrm{w}}^{\circ} \mathrm{RT} \ln \mathrm{N}_{\mathrm{w}}\right) \\
+\mathrm{m} \mu_{\mathrm{s}}^{\circ}+\mathrm{RT} \mathrm{m} \ln \mathrm{m}
\end{aligned}
$$

and the non-ideal (electrical) one

$$
\begin{gathered}
\Delta \mathrm{F}_{\mathrm{el}}^{(\mathrm{T})}=55.5 \mathrm{RT} \ln \mathrm{f}_{\mathrm{w}}+2 \mathrm{RT} m \ln \mathrm{f}_{ \pm} \\
=\Delta \mathrm{F}_{\mathrm{el}(\mathrm{w})}+\Delta \mathrm{F}_{\mathrm{el}(\mathrm{s})}
\end{gathered}
$$

Equation (22) represents the total non-ideal free energy, which can be shown to be given by (PytKowicz et al., 1976)

$$
\Delta \mathrm{F}_{\mathrm{el}}^{(\mathrm{T})}=2 \mathrm{RT} \int_{0}^{\mathrm{m}} \ln \mathrm{f}_{ \pm s} \mathrm{dm}
$$

and the important choice is whether to set $\Delta \mathrm{F}_{\mathrm{L}}$ in equation (15) equal to $\Delta \mathrm{F}_{\mathrm{el}}^{(\mathrm{T})}$ or just to $\Delta \mathrm{F}_{\mathrm{el}(\mathrm{s})}=2 \mathrm{RT} \mathrm{m} \ln \mathrm{f}_{ \pm \mathrm{s}}$.

The choice depends on what we wish to and can represent in a microscopic non-phenomenological model. The term 55.5 RT $\ln \mathrm{a}_{\mathrm{w}}$, which results from the effects of ions on the waters near them, can be taken out so that $\Delta F_{L}$, the lattice free energy, is set equal to $2 \mathrm{RT} m \ln \mathrm{f}_{ \pm \mathrm{s}}$. Still, $\Delta \mathrm{F}_{\mathrm{L}}$ would contain in the term $B_{L}$ the variation in $D_{e}$, the dielectric constant, with $\mathrm{m}$ and $\mathrm{T}$ so that $2 \mathrm{RT} m \ln \mathrm{f}_{ \pm s}$ would not represent purely ion-ion interactions as $D_{e}$ results from ion-water interactions. One can, however, reason that the change in internal $D_{e}$ with concentration in contrast to that of the $D_{e}$ measured with an applied external field, results from the interaction between ions and the waters of hydration of their counter-ions and that this interaction increases as the average interionic distance decreases. If this interaction energy is incorporated into the ionic lattice energy and the effect upon the free energy of the water is neglected then the use of $\Delta F_{L}=$ $2 \mathrm{RT} \ln \mathrm{f}_{ \pm \mathrm{s}}$ is justified.

If, on the other hand, we admit that ionion and ion-water interactions are not strictly separable then it is best to establish a microscopic model of $\Delta F_{L}$ given by equation (23). Then, the parameter $\mathrm{v}$ includes ion-water interactions.

In our earlier work (PYTKowicz et al., 1976) we used this latter approach and now we present a comparison of both methods.

\section{Results}

Values of $\mathrm{L}$ and $\mathrm{v}$ were obtained as follows. At each molality $\mathrm{m}$ values of $\mathrm{L}$ were calculated for arbitrary v's by means of equation (14). The resulting pairs $(\mathrm{v}, \mathrm{L})$ consequently corresponded to minimum values of $\Delta \mathrm{F}_{\mathrm{L}}$, the first condition for an acceptable answer. Then, that pair (v, L) was selected which yielded the second condition, namely, that

$$
\Delta \mathrm{F}_{\mathrm{L}}=\Delta \mathrm{F}_{\text {exp }}=2 \mathrm{RT} \operatorname{mln} \mathrm{f}_{ \pm \mathrm{s}}
$$

or

$$
\Delta \mathrm{F}_{\mathrm{L}}=\Delta \mathrm{F}_{\text {exp }}=2 \mathrm{RT} \int_{0}^{\mathrm{m}} \ln \mathrm{f}_{ \pm \mathrm{s}} \mathrm{dm}
$$

The results are presented for $\mathrm{LiCl}, \mathrm{NaCl}$, and $\mathrm{KCl}$ at $25^{\circ} \mathrm{C}$ in Tables 1 through 3 .

\section{Discussion}

At present we cannot calculate $\mathrm{v}$ from first principles but use it as an adjustable parameter, a common feature of activity coefficient models. This happens because BAHE (1972), PITZER (1973), and Desnoyers and Conway (1964) who treated ion-dielectric gradient interactions, hardcore repulsions, and ion-cavity interactions respectively justified their terms on theoretical 
Table 1. Values of $L, v$, and $\Delta F_{L}$ for LiCl at $25^{\circ} \mathrm{C} . \Delta F_{L}$ is in ergs.

\begin{tabular}{|c|c|c|c|c|c|c|}
\hline \multirow[b]{2}{*}{$\mathrm{m}$} & \multicolumn{3}{|c|}{ Equation (24) } & \multicolumn{3}{|c|}{ Equation (25) } \\
\hline & L & $\mathrm{v}$ & $\Delta \mathrm{F}_{\mathrm{L}}$ & L & $\mathbf{v}$ & $\Delta \mathrm{F}_{\mathrm{L}}$ \\
\hline 0.0001 & 0.5660 & 18.358 & $-5.683 \times 10^{4}$ & 0.5203 & 17.95 & $-3.840 \times 10^{4}$ \\
\hline 0.001 & 0.7080 & 9.377 & $-1.741 \times 10^{6}$ & 0.6580 & 9.017 & $-1.185 \times 10^{6}$ \\
\hline 0.01 & 0.8410 & 5.081 & $-4.952 \times 10^{7}$ & 0.7975 & 4.777 & $-3.463 \times 10^{7}$ \\
\hline 0.1 & 0.9330 & 2.920 & $-1.177 \times 10^{9}$ & 0.9048 & 2.683 & $-8.709 \times 10^{8}$ \\
\hline 0.5 & 0.9650 & 1.983 & $-9.084 \times 10^{9}$ & 0.9420 & 1.771 & $-6.561 \times 10^{9}$ \\
\hline 1.0 & 0.9730 & 1.668 & $-2.092 \times 10^{10}$ & 0.955 & 1.427 & $-1.363 \times 10^{10}$ \\
\hline
\end{tabular}

Table 2. Values of $\mathrm{L}, v$, and $\Delta F_{L}$ for $\mathrm{NaCl}$ at $25^{\circ} \mathrm{C}$.

\begin{tabular}{|c|c|c|c|c|c|c|}
\hline \multirow[b]{2}{*}{$\mathrm{m}$} & \multicolumn{3}{|c|}{ Equation (24) } & \multicolumn{3}{|c|}{ Equation (25) } \\
\hline & $\mathbf{L}$ & $\mathrm{v}$ & $\Delta \mathrm{F}_{\mathrm{L}}$ & L & $\mathrm{v}$ & $\Delta \mathrm{F}_{\mathrm{L}}$ \\
\hline 0.0001 & 0.5670 & 18.36 & $-5.731 \times 10^{4}$ & 0.5230 & 17.95 & $-3.840 \times 10^{4}$ \\
\hline 0.001 & 0.7090 & 9.385 & $-1.754 \times 10^{6}$ & 0.6585 & 9.020 & $-1.190 \times 10^{6}$ \\
\hline 0.01 & 0.8440 & 5.106 & $-5.042 \times 10^{7}$ & 0.7990 & 4.786 & $-3.504 \times 10^{7}$ \\
\hline 0.1 & 0.9370 & 2.963 & $-1.235 \times 10^{9}$ & 0.9093 & 2.715 & $-9.110 \times 10^{8}$ \\
\hline 0.5 & 0.9680 & 2.022 & $-9.554 \times 10^{9}$ & 0.9530 & 1.858 & $-7.582 \times 10^{9}$ \\
\hline 1.0 & 0.9730 & 1.668 & $-2.092 \times 10^{10}$ & 0.9633 & 1.562 & $-1.766 \times 10^{10}$ \\
\hline
\end{tabular}

Table 3. Values of $L, v$, and $\Delta F_{L}$ for $\mathrm{KCl}$ at $25^{\circ} \mathrm{C}$.

\begin{tabular}{|c|c|c|c|c|c|c|}
\hline \multirow[b]{2}{*}{$\mathrm{m}$} & \multicolumn{3}{|c|}{ Equation (24) } & \multicolumn{3}{|c|}{ Equation (25) } \\
\hline & $\mathbf{L}$ & $\mathrm{v}$ & $\Delta \mathrm{F}_{\mathrm{L}}$ & L & $\mathrm{v}$ & $\Delta \mathrm{F}_{\mathrm{L}}$ \\
\hline 0.0001 & 0.5670 & 18.37 & $-5.731 \times 10^{4}$ & 0.5205 & 17.95 & $-3.848 \times 10^{4}$ \\
\hline 0.001 & 0.7090 & 9.386 & $-1.754 \times 10^{6}$ & 0.6585 & 9.022 & $-1.192 \times 10^{6}$ \\
\hline 0.01 & 0.8460 & 5.123 & $-5.127 \times 10^{7}$ & 0.8005 & 4.796 & $-3.545 \times 10^{7}$ \\
\hline 0.1 & 0.9410 & 3.010 & $-1.296 \times 10^{9}$ & 0.9128 & 2.742 & $-9.443 \times 10^{8}$ \\
\hline 0.5 & 0.9740 & 2.116 & $-1.067 \times 10^{10}$ & 0.9595 & 1.924 & $-8.335 \times 10^{9}$ \\
\hline 1.0 & 0.9810 & 1.798 & $-2.480 \times 10^{10}$ & 0.9723 & 1.665 & $-2.064 \times 10^{10}$ \\
\hline
\end{tabular}

grounds but obtained numerical values by curvefitting for their individual effects. Thus, we do not know at present what weight to attach to these various phenomena when they are considered simultaneously.

In a sense, our critique of the Debye-Hückel theory can be extended to the specific interaction models because the theory appears in them in the form of the Guntelberg equation. This means that the Boltzman distribution, derived for non-interacting particles, is applied to a problem the very essence of which is strong coulombic interactions. We should add that, when the non-specific interaction term is given a proper form rather than the Guntelberg one, there will be no reason to question the theoretical validity of specific interaction models which, at present, are of value in a semiempirical sense.

The original cluster integral method of Meyer, when applied to non-electrolyte solutions, was rigorous because of the weak interac- tions present in such solutions. The extension to electrolyte solutions involves the use of the Boltzman distribution for clusters of ions when the configurational integral is used. This detracts from the rigorous theoretical validity of the method to an unknown extent but does not invalidate the fine practical results obtained from the empirical fit of the Gurney term.

We do not wish to claim that our model is superior to other ones but rather that it is one which deserves careful consideration as we use the Boltzman law in a valid manner. Indeed, we do not need it at all for the calculation of $v$ and $\mathrm{L}$ from experimental data and use it properly in the theory of $\mathrm{v}$ as referring to independent states (given by possible $\mathrm{v}, \mathrm{L}$ pairs) which are members of a micro-canonical ensemble.

In examining Tables 1 through 3 one observes that the degree of order $\mathrm{L}$ increases with the molality $\mathrm{m}$ as is expected due to the greater energy of interaction when the ions are closer together which is reflected in the increase of 
the absolute values of $\Delta F_{L}$. The parameter $v$ decreases with $\mathrm{m}$ as is expected from the earlier discussion because $r_{\mathrm{o}} / \overline{\mathbf{r}}_{\mathrm{x}}$ should decrease towards unity with increasing $\mathrm{m}$.

The effect of replacing equation (25), which is exact as it contains no arbitrary separation of ion-ion and ion-water interactions, by the simpler equation (24) is slight upon $L$ and $v$ as can be seen from Tables 1 through 3 . This is as should be because the degree of order and the fluctuations given by $r_{0} / \bar{r}_{x}$ should not be sensitive to ion-water interactions. The difference $\Delta \mathrm{F}_{\mathrm{L}}$ obtained from the two equations results from the removal of $55.5 \mathrm{RT} \ln \mathrm{f}_{\mathrm{w}}$ from equation (24).

Of course, our model will lead to the correct activity coefficient of the salt because of the curve-fitting procedure used for the calculation of $\Delta \mathrm{F}_{\mathrm{L}}$ but our purpose at this stage is explanatory rather than predictive.

We should point out that nothing in our model forces $\mathrm{L}$ to be between 0 and 1 and to tend to 1 as the concentration increases. The fact that this turns out to be the case when we fit the model to experimental data is an independent test of the validity of our method.

Acknowledgments-This research was supported by the Oceanography Section of the National Science Foundation and by the Office of Naval Research Contract N00014-76-C-0067.

\section{REFERENCES}

BAHE, L. W. (1972) Structure in concentrated solutions of electrolytes. Field-dielectric - gradient forces and energies. J. Phys. Chem. 76, 1062-1071.

DEBYE, P. and HüCKEL, E. (1923) Zur Theorie der Elektrolyte. Phys. Z. 24, 185-206.

DESNOYERS, J. E. and CONWAY, B. E. (1964) Activity coefficients of electrolytes at intermediate concentrations and the "cube-root" law. J. Phys. Chem. 68, 2305-2311.

FrANK, H. S. and THOMPSON, P. T. (1959) Fluctuations and the limit of validity of the Debye-Hückel theory. J. Chem. Phys. 31, 1086-1095.

KITTEL, C. (1959) Introduction to solid state physics. John Wiley and Sons, New York, 617 pp.

PAULING, L. (1960) The nature of the chemical bond, 3rd ed. Cornell University Press, Ithaca, $450 \mathrm{pp}$.

PITZER, K. S. (1973) Thermodynamics of electrolytes. I. Theoretical basis and general equations.

PYTKowiCZ, R. M., JOHNSON, K. and CURTIS, C.(1976) A dynamic partial order model of electrolyte solutions. Oregon State University, School of Oceanography Tech. Rept., Corvallis, Oregon, 102 pp.

PYTkowicz, R. M. and KeSTER, D. R. (1969) Harned's rule behavior of $\mathrm{NaCl}-\mathrm{Na}_{2} \mathrm{SO}_{4}$ solutions explained by an ion-association model. Am. J. Sci. 267, 217-229.

ROBINSON, R. A. and STOKES, R. H. (1959) Electrolyte solutions. Butterworths Publications Ltd., London, $571 \mathrm{pp}$. 